Recepción: 16/10/2014

Evaluación: 20/10/2014

Aprobación: 11/12/2014

Artículo de Reflexión

\title{
LAS MISIONES EXTRANJERAS Y SU INFLUENCIA EN LA EDUCACIÓN AGRÍCOLA SUPERIOR EN COLOMBIA
}

\author{
Francisco Javier Torres Martínez \\ Universidad de Nariño \\ Grupo de Investigación: PISIL. Educación Ambiental \\ franjatm@hotmail.com
}

\begin{abstract}
RESUMEN
Este artículo tiene como marco de referencia la Tesis Doctoral en Ciencias de la Educación/RUDECOLOMBIA: "El Programa de Ingeniería Agronómica de la Universidad de Nariño: Análisis, balance y prospectiva: 1962-2012", que examina, entre otros temas, la influencia que ejercieron las misiones extranjeras en los ámbitos de la educación, inicialmente de Europa (Alemania, Bélgica Italia y Francia), y posteriormente de los Estados Unidos, mediante las fundaciones Kellogg, Rockefeller y Ford, y la Alianza para el Progreso. La incursión de programas agrícolas se suscitó, después de los años cincuenta del siglo XX, en las Universidades de Nariño, Tolima y Caldas, entre otras, y fue evidente el dominio que ejercieron estas misiones en los currículos agrícolas en Colombia y en Latinoamérica bajo el patrón de la Revolución Verde. Adicional a la ayuda financiera que fortaleció la Educación Agrícola Superior en Colombia, también significó el establecimiento de modelos de desarrollo, sustentados en el uso intensivo de insumos agroquímicos, con enormes efectos socioeconómicos, que apenas hoy se dimensionan.
\end{abstract}

Palabras clave: Sector primario, Currículo, Plan de estudios, Programa de Ingeniería Agronómica, Universidad de Nariño. 


\title{
FOREIGN MISSIONS AND THEIR INFLUENCE ON AGRICULTURAL HIGHER EDUCATION
}

\begin{abstract}
This article is based on the doctoral thesis in Educational Sciences"RUDECOLOMBIA" The Program of Agricultural Engineering of the University of Nariño: Analysis, balance and foresight: 1962-2012," which examines, among other things, the influence of foreign missions on the areas of education, initially in Europe (Germany, Belgium, Italy and France), and later in the United States, through the Kellogg, Rockefeller and Ford foundations, and the Alliance for Progress. The incursion of agricultural programs arose after the fifties of the twentieth century, at the University of Nariño, Tolima and Caldas, among others, and the dominance exerted by these missions on agricultural curricula in Colombia and Latin America under the pattern of the Green Revolution was evident. Besides the financial assistance which strengthened the Higher Agricultural Education in Colombia, this movement also meant the establishment of development models, supported by the intensive use of chemical inputs, with enormous socioeconomic effects that are sized just today.
\end{abstract}

Keywords: Primary sector, Curriculum, Program of Agricultural Engineering, University of Nariño.

\section{AS MISÕES EXTRANGEIRAS E SUA INFLUÊNCIA NA EDUCAÇÃO AGRÍCOLA SUPERIOR NA COLOMBIA}

\begin{abstract}
RESUMO
Este artigo tem como enquadramento a Tese Doutoral em Ciências da Educação/RUDECOLOMBIA: "O Programa de engenharia Agronômica da Universidade de Nariño: Análise, equilíbrio e visão: 1962-2012", que examinando, entre outras questões, a influência exercida por missões extrangeiras na área da educação, inicialmente da Europa (Alemanha, Bélgica Itália y França), e mais tarde dos Estados Unidos, por meio das fundações Kellogg, Rockefeller y Ford, e da Aliança para o Progresso. A incursão de programas agrícolas surge, depois dos anos cincuenta do século XX, nas Universidades de Nariño, Tolima e Caldas, entre outras, que evidência o dominio que exercido por estas missões nos currículos agrícolas na Colômbia e na Latinoamérica sob o patrono da Revolução Verde. Adicional ao apoio financiero que fortificou a Educação Agrícola Superior na Colombia, também significou o estabelecimento de modelos de desenvolvimento, suportados no uso intensivo de insumos agroquímicos, com enormes efeitos socioeconómicos, são dimensionados apenas hoje.
\end{abstract}

Palavras chaves: Setor primário, Currículo, Plano de estudos, Programa de Engenharia Agronômica, Universidade de Nariño. 


\section{INTRODUCCIÓN}

Las misiones extranjeras se establecieron en Colombia y otros países latinoamericanos en diferentes épocas, principalmente desde los inicios del siglo XX, y desempeñaron un papel importante mediante diagnósticos y recomendaciones de políticas para estimular el desarrollo regional, apoyado en modelos hegemónicos importados. Entre otros temas, y de manera particular el tema educativo colombiano, albergó un marcado dominio en los diseños curriculares y los Planes de estudio de los programas de Educación Agrícola Superior en Colombia.

Es evidente la influencia de las misiones europeas desde principios del siglo XX, hasta 1940; no obstante, después de finalizado el conflicto bélico mundial en 1945, y derivado de los resultados militares, los Estados Unidos se posicionaron y fortalecieron política y económicamente en el ámbito mundial. En adelante, cobran importancia las misiones norteamericanas en el direccionamiento de la enseñanza de las Ciencias agrícolas y con diversos resultados, pero con un objetivo en común, que pretendía incorporar la educación agrícola como dinamizador del desarrollo, mediante estrategias desarrollistas, como la tecnificación del minifundio, el uso drástico de fertilizantes químicos y el control de plagas y enfermedades en los cultivos.

De esta manera, organismos de carácter internacional, como la Organización de Estados Americanos (OEA), la Organización de las Naciones Unidas para la Alimentación y la Agricultura (FAO) y las fundaciones Kellogg, Rockefeller y Ford fueron determinantes en la implementación de Planes de estudios de carácter técnico, la financiación de infraestructura, la capacitación de docentes, la publicación de material bibliográfico y el diseño de sistemas de investigación y transferencia de tecnología hacia los productores rurales.

Con el propósito de aportar elementos adicionales que permitan comprender en mejor forma la influencia que ejercieron estos organismos internacionales sobre la educación y el desarrollo del país, a continuación se describen y analizan las características más relevantes de estas misiones internacionales, que direccionaron los Planes de estudio de programas agrícolas en la Educación Superior.

\section{CONNOTACIONES GENERALES DE LA ACTIVIDAD AGRÍCOLA EN COLOMBIA}

El interés por los recursos naturales fue manifiesto desde tiempos inmemoriales; no obstante, la conquista del territorio americano le imprimió otros vínculos al uso y disposición de estas riquezas, entre otros, aspectos de carácter económico y político determinantes para aquella época en el viejo continente, 
circunstancias que promovieron la creación de verdaderas empresas para el diagnóstico y estudio de estos recursos y, durante el reinado de Carlos IV, tuvieron lugar en la Nueva España varios acontecimientos científicos, como la Expedición de Circunnavegación Mundial (en el periodo de 1788 a 1794) y la Expedición Botánica (1783-1803) ${ }^{1}$, que condujeron al desarrollo de transcendentales avances económicos y sociales. De manera complementaria, otros sucesos alcanzaron especial injerencia en el desarrollo agrícola y el fortalecimiento de la economía en los territorios descubiertos ${ }^{2}$.

Posteriormente, en los inicios de la época republicana, es importante el aprovechamiento de especies agrícolas, entre otras, el café, que fue el soporte fundamental de la economía por varias décadas. En 1824, se iniciaron las exportaciones cafeteras, con la venta de 25.000 sacos $^{3}$; después, otras especies agrícolas, como la quina y el añil, dinamizaron el desarrollo en general.

En concepto de Bejarano ${ }^{4}$, la primera revolución agrícola que precedió la Revolución Industrial ${ }^{5}$ se desplegó en el siglo XVIII, entre otras condiciones por la introducción de prácticas para el manejo de los suelos de uso agrícola, mientras que la segunda revolución agrícola tiene lugar después de 1850, principalmente en Alemania, Inglaterra, Francia, Dinamarca, Rusia, España y Estados Unidos, que consistió en la aplicación de la ciencia a la agricultura, mediante la utilización de implementos mecánicos y el uso de fertilizantes químicos; esta segunda revolución igualmente tuvo estrecha relación con la educación agrícola y la aparición de medios de comunicación masiva para transformar los hábitos de los agricultores. Aún con estas transformaciones en el siglo XIX, los alcances de las dos revoluciones agrícolas en Colombia fueron mínimos y notorios el atraso técnico de la agricultura neogranadina ${ }^{6}$.

1. La Expedición Botánica tenía por objeto la recolección y nomenclatura de plantas desconocidas, su dibujo y ordenamiento científico. Estudiar el reino animal y los minerales, observar los fenómenos astronómicos y describir la geografía del Virreinato; además, preparar los materiales para formar colecciones y herbarios. Para realizar estos trabajos, se organizaron grupos de trabajo, bajo la supervisión de José Celestino Mutis. http://www.bibliotecanacional.gov.co/recursos_user/exposicionesvirtuales/ expedicion_botanica/pdf/expedicion_botanica.pdf (23 de Julio de 2012).

2. Víctor Patiño. Esbozo histórico agropecuario del periodo republicano en Colombia (Bogotá: Caro y Cuervo, 2002), 20.

3. El café, desde finales del siglo IXX, representó un renglón importante de la economía colombiana, hasta mediados del siglo XX, y uno de los productos principales de exportación. Daniel Macías. 50 años de la Agronomía en Colombia (Bogotá, Duplolito, 1978), 14.

4. Jesús A. Bejarano. Ensayos de historia agraria colombiana (Bogotá: Fondo Editorial CEREC, 1987), 116120.

5. La Revolución Industrial se define como el proceso de transformación económico, social y tecnológico, en la segunda mitad del siglo XVII, que se inició en Gran Bretaña y se extendió a Europa y luego a Estados Unidos hasta 1840. Las transformaciones que se dieron fueron sustanciales, al pasar de una economía rural a una industria basada en la mecanización.

6. Bejarano, Ensayos de historia agraria colombiana, 128. 
Valencia y Acevedo ${ }^{7}$ coinciden con estas apreciaciones y argumentan que, desde la época colonial y durante el siglo XIX, es notable el rezago de la agricultura en los territorios que actualmente ocupa Colombia, con excepción de algunas innovaciones relacionadas con especies agrícolas de pastos, el uso del alambre de púas y elementales mejoras técnicas en plantaciones cafeteras; también se valoran, durante la época citada, los esfuerzos institucionales por establecer elementos de la segunda revolución agrícola ${ }^{8}$. No obstante, desde mediados del siglo XIX, también se pretendía transformar la agricultura, con el apoyo de las ciencias, las técnicas experimentales y la Educación Superior como promotor del desarrollo del país.

En 1913, mediante la Ley $25^{9}$, se creó el Ministerio de Agricultura, que dinamizó el sector agrícola; además, otros acontecimientos influyeron en el desarrollo, como la puesta en marcha del Canal de Panamá y la llegada al Valle del Cauca del Ferrocarril del Pacífico, en 1915, circunstancias que propiciaron el tránsito de la agricultura de subsistencia a las explotaciones comerciales de caña de azúcar, cacao, tabaco y otros productos.

Para el primer cuarto del siglo XX, sigue siendo importante para el país la actividad cafetera y su acelerado impulso fue posible por la disponibilidad de mano de obra, que representó, en su momento, el 50\% de las exportaciones, comportamiento que se mantuvo hasta después de $1924^{10}$, condiciones que permitieron, a partir de 1925, aumentar la producción de materias primas y alimentos en gran parte del territorio nacional, lo que se mantuvo hasta mediados del siglo XX.

Sin embargo, después de 1950, el crecimiento de la agricultura comercial no fue suficiente para satisfacer la demanda interna y se recurrió a las importaciones de alimentos. Según De Pombo ${ }^{\mathbf{1 1}}$, la actividad agrícola no fue una alternativa para la generación de divisas y se importaron trigo, maíz y frijol.

7. Néstor Valencia y Álvaro Acevedo. Políticas agrarias del estado-nación colombiano, Los inicios de la educación agrícola superior en el Valle del Cauca. En: Diana Soto, Justo Cuño y Jorge Tomas (eds.). La construcción de la Nación Iberoamericana. Siglos XIX a XXI (Bogotá: VENDIMIA, 2010), 214.

8. Néstor Valencia L. Génesis e irrupción de la agricultura científica: La educación agrícola superior en el Valle del Cauca, Colombia, 1910-1946 (Pereira: Universidad Tecnológica de Pereira/RUDECOLOMBIA, 2006), 55-56 (Tesis doctoral).

9. La Ley 25, de octubre 8 de 1913, dividió en ocho Ministerios el Despacho Administrativo del Gobierno colombiano: Gobierno, Relaciones Exteriores, Hacienda, Guerra, Instrucción Pública, Agricultura y Comercio, Obras Públicas y Tesoro, que iniciaron funciones el 7 de agosto de 1914.

10. Aline Helg. La educación en Colombia 1918-1957 (Bogotá: Fondo Editorial CEREC, 1987), 89-90.

11. Joaquín de Pombo. Dinámica del sector agrícola colombiano. En: Francisco E. Thoumi et al (eds.). La economía colombiana, temas de comercio y desarrollo (Bogotá: Banco de la República, 1995), 225. 
Para los inicios de la dictadura militar ${ }^{12}$, entre 1953 y 1955, se registró una mejoría de las condiciones, originada principalmente por los precios internacionales del café ${ }^{13}$, lo que da lugar a una etapa corta de progreso de la agricultura capitalista; no obstante, su comportamiento fue irregular y, entre 1955 y 1957, la producción agrícola del país disminuyó. De manera paralela, este contexto deficitario de producción desencadenó una importación de alimentos y materias primas. Cabe anotar que las importaciones a gran escala de cereales aún persisten, y en mayores proporciones, en virtud de modelos aperturistas a partir de la décadas de los años ochenta y noventa del siglo anterior.

Después de estos indicadores adversos, los escenarios del país fueron más propicios para el desarrollo económico al amparo de la influencia capitalista y se instauraron estímulos para el fomento del sector agrícola, mediante reducción de los impuestos a los productores de cacao, caucho, palma africana y otras especies agrícolas de largo plazo. En ese mismo sentido, la Ley 26 de 1959 amplió el acceso al crédito agrícola a otras actividades, como los cultivos de largo plazo (palma africana, frutales, especies maderables, café, entre otros).

En 1961, tiene lugar un acontecimiento de gran controversia, como fue la Ley $135^{\mathbf{1 4}}$, que constituyó el Instituto Colombiano de la Reforma Agraria (INCORA), con el propósito de devolver la titulación de tierras a los productores rurales, principalmente en zonas de violencia. Posteriormente, el marco legal se amplió mediante la expedición de leyes para el fomento del sector primario; entre otras, la Ley 5 de 1973, que reglamentó el Fondo Financiero Agropecuario (FFAP); la Ley 85 de 1985, que impulsó la comercialización, y, más recientemente, la Ley 16 de 1990, que reglamentó el Fondo para el Financiamiento del Sector Agropecuario (FINAGRO).

Otros mecanismos de protección a la producción nacional se contemplan en la Constitución Política de 1991 y la Ley 101 de 1993, que reorganizan medidas de protección a la producción; entre otras, subsidios a la producción agrícola, incentivos a la Capitalización Rural (ICR), Incentivo Forestal y el Fondo Agropecuario de Garantías (FAG). No obstante, la aplicación más agresiva de modelos de tendencia eminentemente neoliberal, como la apertura económica desde finales de los años ochenta, ha llevado a miles de pequeños productores a la

12. Durante el periodo de la dictadura militar, entre 1953 a 1957, el General Gustavo Rojas Pinilla (19001975) fue Presidente de Colombia.

13. En el periodo de 1953 a 1957, el café colombiano se cotizó, en promedio a U\$ 0,90/libra. Ver: Hernando Agudelo. Cuatro etapas de la inflación en Colombia (Bogotá: Tercer Mundo, 1966), 174.

14. La Ley 135 de diciembre 13 de 1961, durante la segunda presidencia de Alberto Lleras Camargo (19581962), incluyó entre otros temas, los Procuradores Agrarios, el Fondo Nacional Agrario, la Extinción del dominio sobre tierras incultas, las Unidades Agrícolas Familiares, reglamentación sobre baldíos, adecuación de tierras, parcelarios y minifundio. 
quiebra económica, originada por la importación masiva de cereales, carnes y productos lácteos.

Para concluir este breve examen de la actividad agropecuaria en Colombia, es necesario mencionar los controvertidos Tratados de Libre Comercio (TLC), merced a las tendencias de la economía global, propuestos a nivel mundial por los organismos supranacionales, como el Banco Mundial. Concordante con estas políticas de globalización, el país ha suscrito convenios y tratados de comercio en los últimos años con varios países y bloques económicos, entre los más destacados con Estados Unidos, Corea, Centro América, la Unión Europea y con países del Pacífico (México, Chile y Perú). Sin embargo, en concepto de la mayoría de los gremios de la producción agrícola, han expuesto al país a las condiciones de mercados internacionales, en los cuales la producción se ampara en esquemas de subsidios, que hacen imposible que compitiesen en condiciones favorables los pequeños y medianos productores rurales.

\section{EFECTOS Y CONSECUENCIAS DE LA REVOLUCIÓN VERDE}

El rápido análisis de los sucesos en torno a la actividad agropecuaria, en Colombia, no estaría completo, si antes no se advierte una rápida mirada a un acontecimiento que tuvo sus inicios después de la segunda mitad del siglo anterior y que, en mayor o menor proporción, afectó económica, cultural y políticamente al país, como fue la Revolución Verde, un modelo desarrollista concebido y apoyado por los Estados Unidos, que, sin duda, se vinculó estrechamente a las pretensiones de las misiones extranjeras.

Colombia no fue ajena a estos lineamientos agrícolas de carácter regional y mundial, y adoptó estos postulados desarrollistas, con lo que ha obtenido no sólo sus beneficios, sino también sus adversas consecuencias. León ${ }^{15}$ las describe así:

El modelo de desarrollo agrario seguido por Colombia en las últimas décadas, basado en los postulados de intensificación de la productividad proveniente de la Revolución Verde, ha incidido en la aparición de procesos de degradación de los ecosistemas, marginalidad rural y desigualdad social, que afectan gran parte de este sector productivo.

De hecho, los principios fundamentales de la Revolución Verde se inspiraron en el mejoramiento de la productividad mediante la utilización de variedades agrícolas mejoradas de maíz, trigo y arroz, con lo que se intentaba el incremento sustancial de los rendimientos por hectárea, pero, al mismo tiempo, la adopción

15. LEÓN, Tomás. Medio ambiente, tecnología y modelos de agricultura en Colombia (Bogotá: Ecoe Ediciones, 2007), 61. 
inevitable de otras prácticas, como el uso de grandes cantidades de agua, fertilizantes y plaguicidas ${ }^{16}$.

En Colombia, este modelo de desarrollo se intensificó entre 1960 y 1978, principalmente para cultivos comerciales de algodón, arroz, sorgo y caña de azúcar, lo que ha dado lugar al incremento de las superficies sembradas, el uso intensivo de maquinaria y fertilizantes. Particularmente en especies de clima frío, se registró aumento importante en los rendimientos de maíz, que se incrementaron de 1200 a $2100 \mathrm{~kg} / \mathrm{ha}$, indicadores que evidencian un mejoramiento tecnológico mediante fertilización, el uso de variedades nuevas y el control de plagas y enfermedades ${ }^{17}$.

Por otra parte, los efectos de los agroquímicos han suscitado, en el país en los últimos años, fuertes controversias en torno a la sostenibilidad de la agricultura y el medio ambiente. Sin embargo, subsisten discrepancias alrededor del tema, sobre las definiciones y énfasis que deban tener las políticas para alcanzar la sostenibilidad, y falta mucho para articularla como problema del desarrollo ${ }^{\mathbf{1 8}}$.

Por lo demás, los efectos de los agroquímicos son de diversa índole, al considerar no sólo el comportamiento agronómico de especies agrícolas, sino también las consecuencias sociales y económicas evidentes acarreadas en la mayoría de países que apadrinaron este patrón de explotación, circunstancias que han derivado en un amplio debate, entre detractores y protectores de estos estándares, y se ha visto, por los defensores, como un milagro que ha evitado hambrunas de la población pobre, además de la producción de variedades agrícolas más resistentes a factores de clima, plagas y enfermedades, mientras que, desde el punto de vista de los detractores, ha significado el uso indiscriminado de insumos agroquímicos, deterioro de suelos, contaminación y explotación de los pobres, en beneficio de unos pocos.

\section{LA EDUCACIÓN AGRÍCOLA EN COLOMBIA}

La educación agrícola tiene un impulso importante en Colombia durante el período de la República Liberal (1930-1946), como consecuencia directa de las reformas educativas emprendidas inicialmente por el presidente Enrique Olaya Herrera (1930-1934); al respecto, Triana ${ }^{19}$ comenta: “La educación vocacional

16. El ciclo que abarca estos acontecimientos se ubica entre 1940 y 1970 , y tiene como promotor principal al ingeniero agrónomo estadounidense Norman Borlaug (1914-2009), considerado el padre de la Revolución Verde, que, entre otros reconocimientos, obtuvo el Premio Nobel de Paz en 1970.

17. León, Medio ambiente, tecnología y modelos de agricultura en Colombia, 74.

18. Jesús Antonio Bejarano. Economía de la Agricultura (Bogotá: TM Editores/Universidad Nacional/IICA, 1998), 299.

19. Alba Nidia Triana. Escuelas Normales Rurales, Agropecuarias y de Campesinas en Colombia: 1934-1974. En: Revista Historia de la Educación Colombiana, No. 13 (2010), 203. 
de agricultura aparece en Colombia con el nacimiento de la Escuela Normal para Mujeres (1934), creada en la República Liberal, como respuesta a la importante campaña política de vincular la sociedad rural a la modernización".

De otro lado, los Institutos Técnicos Agrícolas (ITA) inician sus labores en el país después de 1940, para ofrecer un Plan de estudios en dos etapas: una, para cursos cortos, y otra científica, que incluía cinco años de educación primaria y cuatro años de educación secundaria, para obtener el título de Instructores Agrícolas. De acuerdo a Gracia ${ }^{20}$, la educación vocacional agrícola se fortaleció en Colombia mediante la Ley 164 y el Decreto 543 de 1941, con el propósito de elevar el nivel de vida de la población campesina mediante las prácticas agrícolas propias de cada región, en Escuelas vocacionales agrícolas.

Posteriormente en 1943 el Congreso de la República mediante la Ley 43 transformó a las Escuelas industriales en Institutos Técnicos Superiores y por Decreto Ley 78 del mismo año, las Granjas Vocacionales Agrícolas pasaron a ser las Normales Agrícolas ${ }^{21}$. Más adelante, a partir de 1946, con el régimen conservador en el poder, tiene lugar la violencia política y, con ello, la persecución a las ideas liberales que se habían instaurado en la hegemonía anterior; se nombran profesores con ideas conservadoras, se intensifica la discriminación en contra de los liberales y, así mismo, se afecta el nivel de calidad de los maestros rurales ${ }^{22}$.

Las Normales Agrícolas de Paipa, Lorica y Buga funcionaron hasta 1966 y luego se transformaron en los ITA. No obstante, en 1976, los ITA nuevamente regresan al Ministerio de Educación Nacional, con un Plan de estudios que incluye seis años de bachillerato en la modalidad agropecuaria y la intermedia profesional.

En este sentido, la modernización de la educación agropecuaria, en el país, no fue el resultado de un análisis sectorial, sino de la necesidad de cumplir los requerimientos y obligaciones con las políticas para el desarrollo de América Latina, que justamente impulsaban las misiones extranjeras. En este sentido, los ITA cumplieron funciones de modernización y transferencia de tecnología,

20. Roberto Gracia. La educación agrícola vocacional en Colombia (Bogotá: Instituto Interamericano de Ciencias Agrícolas (OEA), Instituto para el Fomento de la Educación Superior (ICFES) y Programa de Estudios para Graduados Universidad Nacional de Colombia/ICA, 1977), 9.

21. Reseña histórica de los Institutos Técnicos Agrícolas. En: Instituto Técnico Agrícola de Guadalajara de Buga (Valle del Cauca), http://www.ita.edu.co/index.php/2013-10-18-14-31-32/resena-historica (14 de abril de 2013).

22. Alba Nidia Triana. Formación de maestros rurales colombianos 1946-1994. En: Revista Historia de la Educación Latinoamericana. Volumen 14, No 18 (2012), 97. 
similar a los Institutos de Educación Media (INEM), que fueron la reproducción del modelo de las Escuelas Comprensivas Americanas ${ }^{23}$.

Por otra parte, el Servicio Nacional de Aprendizaje (SENA) ${ }^{24}$ estableció, a partir de 1957, actividades de formación profesional para trabajadores jóvenes y adultos de la industria, el comercio, la agricultura, la minería y la ganadería. En 1958, surgió el programa de Capacitación para la Participación Campesina (CAPACA), dirigido a líderes campesinos, indígenas y comunidades beneficiarias de la Reforma Agraria.

Durante el período del Frente Nacional (1958-1974) ${ }^{25}$, se promulgaron reformas educativas y pedagógicas a favor de los grupos de población rural más vulnerable, y se recibió ayuda extranjera para vincular al país al desarrollo económico y social. Por supuesto, las intenciones de estas y otras tantas reformas fueron más optimistas que sus resultados a lo largo de la historia del país; inclusive, aún son evidentes, mediante la radicalización de las desigualdades económicas, culturales y sociales entre lo rural y lo urbano.

Otro modelo de educación rural en el país fue el proyecto de la Escuela Nue$\mathrm{va}^{26}$, a partir de 1982, diseñado para zonas rurales de baja densidad de población, y se puede calificar de exitoso en el sentido de que ha contribuido a solucionar las necesidades de la educación primaria rural. Mientras tanto, la gestión del Banco Mundial se concentraba en la educación técnica y vocacional, mediante préstamos otorgados a Colombia en 1968, 1970 y 1982, que permitieron establecer los INEM en varias ciudades de Colombia ${ }^{27}$. En la ciudad de Pasto, inició actividades en 1970, y fue una novedad el sistema de titulación de bachilleres mediante las modalidades industrial, comercial, académica y agropecuaria.

23. Triana, Escuelas Normales Rurales, Agropecuarias y de Campesinas en Colombia, 218-219.

24. El Instituto Nacional de Aprendizaje (SENA) se creó durante el Gobierno de la Junta Militar, posterior a la renuncia del General Gustavo Rojas Pinilla, mediante el Decreto-Ley 118, del 21 de junio de 1957.

25. El Frente Nacional fue el periodo de 16 años, comprendido entre 1958 y 1974, que se dio por la coalición política entre los partidos tradicionales colombianos liberal y conservador, en el que se alternaron el poder cada cuatro años y pretendía la reorganización de Colombia, posterior al periodo presidencial del general Gustavo Rojas Pinilla y la transición de la Junta Militar.

26. La Escuela Nueva fue una estrategia impulsada por el Banco Mundial para la educación primaria, desde 1982, y tenía en la época más de 2000 escuelas en áreas rurales, y en 1987 el Plan de desarrollo del Presidente Barco (1986-1990) la incluyó para ampliar la cobertura a todas las áreas rurales del país, y para 1989 había más de 18000 escuelas. Ver: Jesús Duarte. Educación pública y clientelismo en Colombia (Medellín: Facultad de Ciencias Humanas y Económicas, Universidad Nacional de Colombia- Sede Medellín, 2003), 241-244.

27. Duarte, Educación pública y clientelismo en Colombia, 161 y 162. 


\subsection{La educación agrícola superior en Colombia}

La enseñanza formal de las Ciencias agrícolas en Colombia tiene sus inicios a principios del siglo XIX, y se hace efectiva en 1826, mediante la organización de la educación ${ }^{28}$ en todos sus niveles, lo que incluye la enseñanza de las Ciencias agrícolas. Ocampo ${ }^{29}$ asegura que al tema de la educación en Colombia sólo se le asigna una importancia mayor a partir de 1826, con la promulgación de la Ley Republicana de Organización de la Educación; como tal, esta Ley culminó sus efectos en 1843, cuando se aprobó la Constitución Política de la Nueva Granada.

En América Latina, la enseñanza agrícola ya se impartía desde mediados del siglo XIX, con la fundación en 1854 de la Escuela Nacional de Agricultura de San Jacinto $^{30}$ (Chapingo) en México, y a finales del siglo XIX y principios del siglo XX, surgen en varios países Escuelas o Facultades de agronomía; a finales del siglo XIX, ya existían Facultades en México, Brasil, Argentina y Chile.

Mientras tanto, a mediados del siglo XIX se acentúan los esfuerzos institucionales del país por transformar la agricultura mediante la tecnificación ${ }^{31}$, con acciones que conducirían, más tarde, a la institucionalización de la enseñanza ${ }^{32}$ de las Ciencias agrícolas en diversos ámbitos de la vida nacional. Camacho Roldán ${ }^{33}$ describía, para este período, una situación conmovedora del atraso

28. En cumplimiento del Decreto de 18 de marzo de 1826, el interés por la educación en todos los niveles fue constante. Con miras a romper la estructura curricular clásica e incorporar asignaturas en ciencias, se propuso un Plan de estudios para escuelas, colegios y universidades, y se adoptaron importantes medidas para mejorar la educación. Firmado en el Palacio del Gobierno de Colombia, en Bogotá, a 3 de octubre de 1826, por Francisco de Paula Santander, por su Excelencia el Vicepresidente de la República, el Secretario de Estado del Despacho, http://www.banrepcultural.org/blaavirtual/historia/hnuegra/ hnuegra2.htm (14 de abril de 2014).

29. Javier Ocampo. Los orígenes oficiales de las universidades republicanas en la Gran Colombia (18261830). En: Estudios sobre la universidad latinoamericana, de la Colonia al siglo XIX. Diana Soto, Manuel Lucena y Carlos Rincón (Pereira: Universidad Pedagógica y Tecnológica de Pereira, 2002), 160-161.

30. La antigua Escuela Nacional de Agricultura, desde 1854, desarrolló sus actividades en las vetustas edificaciones y terrenos del ex convento de San Jacinto, hoy asimilado a la incontrolable mancha urbana del Distrito Federal; fue trasladada a la ex hacienda de Chapingo en 1923; lleva consigo numerosos episodios históricos, que configuraron el antecedente inmediato de una nueva institución de enseñanza agrícola superior y comprometida con los ideales agraristas enarbolados por la Revolución Mexicana. Ver: Silvia González. Historia de la Hacienda de Chapingo (México: Universidad Autónoma de Chapingo, 1996), 15.

31. Valencia y Acevedo, Políticas agrarias del estado-nación colombiano, 213.

32. Se refiere a los términos de su formalización y regulación, ordenaciones a las que subyacen corrientes de pensamiento, doctrinas e ideales que conciben de diversa forma al ser humano, la función del conocimiento y la misión de la educación. Ver: la Tesis doctoral de Mireya Uscátegui. La institucionalización de la enseñanza del arte en Colombia: Antecedentes y evolución (De la Colonia al siglo XIX) (Pasto: Universidad de Nariño/ RUDECOLOMBIA, 2010), 28.

33. Salvador Camacho Roldán, economista, jurista, editor, periodista (1828-1900). Sus estudios sobre la agricultura, las vías de comunicación y la educación son punto de referencia indispensable para conocer la realidad del país en el siglo XIX; se destacó como uno de los políticos más activos del país. Sus obras publicadas son: Notas de viaje (1890), elaboradas a raíz de su viaje a los Estados Unidos en 1887; Escritos varios (1892-1895) y Memorias, publicadas por primera vez en 1925. En 1882, pronunció, en la Universidad Nacional, su célebre discurso sobre la sociología, http://www.banrepcultural.org/blaavirtual/biografias/ camasal.htm Biblioteca Luis Ángel Arango del Banco de la República (septiembre 23 de 2013). 
tecnológico que padecía el país y, como parte de la solución de este fenómeno, se adelantaron varias acciones, entre ellas la Ley 66 de 1867, que institucionalmente dio origen a la Universidad Nacional de los Estados Unidos de Colombia, que incluyó las escuelas de Derecho, Medicina, Ingeniería, Ciencias naturales, Literatura, Filosofía, Artes y oficios.

Por su parte, Mesa $^{34}$ afirma que la primera Facultad de agronomía surge entre los años 1874 y 1881, ubicada en región del actual Departamento de Cundinamarca. Por aquella época, se registró un inusitado interés por los temas agrícolas, y en la presidencia de Julián Trujillo (1878-1880) surge el Departamento de Agricultura Nacional.

Entre 1870 y 1900, los resultados de la educación agrícola en el país fueron poco alentadores debido al escaso apoyo del Estado; no obstante, posteriores a 1900, se registran otros acontecimientos en torno a la educación agrícola, mediante Leyes y Decretos, entre otros: el "día del árbol" (Decreto 491 de 1904), obligación de la enseñanza agrícola (Ley 25 de 1913), creación del Instituto Nacional de Agronomía (Ley 3 de 1914 y Decreto 123 de 1915), y la Ley 38 de 1914, que fundó el Instituto de Agricultura y Veterinaria que, a la postre, dio origen a la Escuela Superior de Agricultura de Bogotá.

Después de 1910, se introducen en el país variedades de especies agrícolas más rendidoras, que necesitaban más insumos, especialmente fertilizantes y herbicidas; y a los técnicos agrícolas los empiezan a formar con el nuevo modelo, que se centra en el uso masivo de fertilizantes, pesticidas y variedades acondicionadas a este esquema ${ }^{35}$. Una vez se logra institucionalizar la educación agrícola en el país, se contrató, en 1914, al ingeniero agrónomo belga Carlos Denemoustier, para organizar la enseñanza de las Ciencias agrícolas en el país y se formuló el Plan de Educación Agrícola.

Helg $^{36}$ asegura que una iniciativa, con relativo éxito, fue la enseñanza de las prácticas agrícolas en cultivos de café, actividad que más tarde obtuvo el apoyo de la Federación Nacional de Cafeteros, a partir de 1929, con el propósito de aumentar los rendimientos por hectárea mediante la formación de expertos y, de esta manera, la enseñanza agrícola en Colombia empezó a tomar forma.

A principios del siglo XX, tienen importancia los temas agronómicos en la Escuela de Agronomía de la Universidad de Antioquia, y en 1915 se funda, en Bogotá, la Escuela Superior de Agronomía. En 1920, obtienen el título los pri-

34. Daniel Mesa. La agronomía en Colombia (Bogotá: no registra editorial y fecha), 18.

35. Aurelio Suárez. El modelo agrícola colombiano y los alimentos en la globalización (Bogotá: Ediciones Aurora, 2007), 39

36. Helg, La educación en Colombia 1918-1957, 98-101. 
meros Ingenieros agrónomos de este centro de Educación Superior ${ }^{37}$ y se inicia una etapa técnica, cuya intención es conducir al país al desarrollo agrícola. Subsiguientemente, esta Escuela de Educación Superior dio origen a la Facultad de Agronomía de la Universidad Nacional de Colombia.

En concepto de Bejarano ${ }^{38}$, la promulgación de la Ley de Fomento de la Agricultura, de $1926^{39}$, estableció por primera vez la relevancia de la educación agrícola en las políticas de desarrollo, que se hicieron efectivas mediante actividades de crédito agropecuario, programas de extensión agrícola y campañas de diversificación de cultivos y legislación sobre propiedad de tierras.

También se creó el Consejo Nacional de Agricultura, que promovió la investigación, la educación de alto nivel y los servicios de extensión agrícola, con lo que se hacen evidentes gestiones relacionadas con la educación y se fortalecen las Facultades de agronomía y las Estaciones experimentales, que desempañaron una función importante en los avances técnicos de este período ${ }^{\mathbf{4 0}}$.

El Decreto 262 de 1934 dio origen, en Cali, a la Escuela Superior de Agricultura Tropical (ESAT), que posteriormente pasó a ser la actual Facultad de Ciencias Agropecuarias de la Universidad Nacional ${ }^{41}$. La fundación de la ESAT coincide con un nuevo crecimiento económico regional, entre 1934 y 1939; entre otros, el Producto Interno Bruto aumentó 34\%, el sector industrial creció el 78\% y la agricultura el $17 \%{ }^{42}$.

En 1940, el país contaba con tres Facultades de agronomía; no obstante, durante la Segunda Guerra Mundial (1939-1945), la dinámica de las actividades educativas disminuyó y, luego, "sobrevino una explosión en el campo de la educación agrícola”33, que culminaría alrededor de 1960, con la creación de Estaciones experimentales de exploración climatológica.

De acuerdo con Buriticá ${ }^{44}$, después de 1950, la educación agrícola en Colombia evidencia interés por seguir el modelo Land Grant Colleges de enseñanza agrícola de los Estados Unidos. Para la época, se suscitó una gran actividad bo-

37. El 16 de junio de 1920, se titularon los primeros seis ingenieros agrónomos en la Escuela Superior de Agricultura de Bogotá: Arsenio Bermúdez, Isaías Bolívar, Roberto Pedraza, Rafael Lema, Alfonso Romero y Feliz Díaz. Ver: Macías, 50 años de la Agronomía en Colombia, 5.

38. Bejarano, Ensayos de historia agraria colombiana, 186.

39. Ley 74, de noviembre 30 de 1926, durante el gobierno de Miguel Abadía Méndez (1926-1930). Entre otras disposiciones, esta ley organiza un Instituto Agrícola Nacional como base de los Estudios superiores de agronomía y agricultura y centro de investigación y consulta en temas agrícolas.

40. Bejarano, Ensayos de historia agraria colombiana, 187-189.

41. Patiño, Esbozo histórico agropecuario del periodo republicano en Colombia, 61.

42. Entre otras consideraciones, el autor alude al crecimiento económico del Valle del Cauca. Ver: Valencia, Génesis e irrupción de la agricultura científica, 116.

43. Mesa, La agronomía en Colombia, 24.

44. Pablo Buriticá. Las enfermedades de las plantas y su ciencia en Colombia (Medellín: Universidad Nacional de Colombia, 1999), 221-222. 
tánica, influida por el Instituto de Ciencias Naturales de la Universidad Nacional; además, surgen institutos relacionados con el apoyo del sector agropecuario nacional, como el Departamento de Investigaciones Agropecuarias (DIA) ${ }^{45}$, que más tarde pasó a ser el ICA.

Concordante con el acelerado fomento en el campo de la educación agrícola, se crean, en el país, programas académicos agrícolas, y en 1955 ya funcionaban varios, como: Agronomía en la Universidad Nacional de Colombia, Universidad de Caldas, Universidad de Nariño y Universidad del Tolima, y Biología, en la Universidad de los Andes, Universidad de Antioquia y Universidad Pedagógica de Tunja.

Varias regiones del país registran diversos escenarios en la educación agrícola desde principios del siglo XX, y este accionar es más relevante a partir de 1950, derivado de las recomendaciones técnicas y la ayuda financiera de las misiones internacionales para impulsar la educación agrícola (Tabla 1). En los últimos años, también ha sido relevante la incursión de la educación privada, con la que han surgido los programas agrícolas en la Universidad de La Salle, la Universidad de Ciencias Aplicadas y Ambientales (UDCA) y la Universidad Católica de Oriente ${ }^{46}$.

En este mismo sentido, Goyes y Uscátegui ${ }^{47}$ destacan que, después de un atraso significativo que se extendió hasta la mitad del siglo XX, el país experimentó un notable desarrollo de la educación durante los años 60 y 70 del siglo anterior (después del Frente Nacional), y la mayor expansión educativa se desarrolló en forma paralela al crecimiento del país. Concordante con la situación, el Informe del Banco Mundial ${ }^{48}$ confirma esta tendencia y registra que la matrícula en las instituciones de Educación Superior de Colombia se triplicó entre los años de 1960 a 1968, y en 1969 sucedió un nuevo aumento.

45. En concepto de Daniel Macías, “Posiblemente uno de los acontecimientos más importantes para el mundo colombiano, fue la creación del Departamento de Investigaciones Agropecuarias (DIA)”. El DIA se creó por Decreto 962 de 1955, como dependencia autónoma especial para centralizar la investigación agropecuaria encaminada a incrementar la producción agrícola nacional. Ver: Macías, 50 años de la Agronomía en Colombia, 115.

46. Pedro Alarcón y Néstor Romero. Estado actual de los currículos de Ingeniería Agronómica y Agronomía en Colombia (Bogotá: ACOFIA, 2013), api.ning.com/files/.../librocompleto.docx.

47. Las autoras señalan, también, que, para la época, Colombia registraba en su mayoría educación básica, bachillerato dirigido al campo laboral y la Educación Superior se encargaba de formar a la clase dirigente del país. Ver: Isabel Goyes y Mireya Uscátegui. Investigación y pedagogía (Pasto: Universidad de Nariño, 1999), 145.

48. El Banco Mundial elaboró un informe de la Misión Económica enviada a Colombia en 1970. El informe incluye 25 Capítulos que analizan diversos sectores del país, entre los que se destacan: La población, el empleo, el desarrollo, el sector agrario, la pobreza, la educación y los servicios públicos, entre otros. Ver: Banco Mundial. El desarrollo económico de Colombia, Problemas y perspectivas (Bogotá: Biblioteca Banco Popular, 1970), 665. 
Tabla 1. Establecimiento y evolución de algunos Programas de Educación Agrícola Superior en Colombia

\begin{tabular}{|l|l|l|}
\hline \multicolumn{1}{|c|}{ UNIVERSIDAD } & \multicolumn{1}{|c|}{ PROGRAMA } & AÑO \\
\hline Universidad Nacional de & Escuela de Agricultura & 1911 \\
Colombia - Medellín & Escuela de Agricultura Tropical & 1914 \\
& Facultad Nacional de Agronomía & 1938 \\
\hline \multirow{3}{*}{ Universidad Nacional de } & Escuela Superior de Agricultura Tropical & 1934 \\
Colombia - Palmira & Facultad de Agronomía del Valle & 1944 \\
& Facultad de Agronomía de Palmira & 1949 \\
& Facultad de Ciencias Agropecuarias & 1970 \\
\hline Universidad de Caldas & Facultad de Agronomía & 1950 \\
\hline Universidad del Tolima & Facultad de Ingeniería Agronómica & 1956 \\
\hline Universidad Pedagógica y & Facultad de Agronomía & 1960 \\
Tecnológica de Colombia-Tunja & & \\
\hline & Instituto Agronómico Departamental & 1913 \\
Universidad de Nariño & Facultad de Agronomía & 1946 \\
& Facultad Superior de Agronomía & 1958 \\
Instituto Tecnológico Agrícola (ITA) & 1958 \\
\hline Universidad Tecnológica del & Facultad de Ciencias Agrícolas (FACIA) & 1971 \\
\hline Magdalena & Facultad de Ingeniería Agronómica & 1962 \\
\hline Universidad de Córdoba & Facultad de Ingeniería Agronómica & 1964 \\
\hline Universidad de los Llanos & Facultad de Ciencias Agropecuarias & 1970 \\
\hline $\begin{array}{l}\text { Universidad de Ciencias } \\
\text { Aplicadas y Ambientales UDCA } \\
\text { (Privada) }\end{array}$ & Programa de Ingeniería Agronómica & 1983 \\
\hline $\begin{array}{l}\text { Universidad de Cundinamarca, } \\
\text { UDEC }\end{array}$ & Programa de Ingeniería Agronómica & 1969 \\
\hline
\end{tabular}

Fuente: Esta investigación.

Asimismo, cabe anotar que las iniciativas por instaurar programas de Educación agrícola superior en Colombia, en ciertas regiones del país, no propiamente surgieron directamente de las universidades ya establecidas; por el contrario, se iniciaron en las Asambleas departamentales, más como hechos de connotación política que académica, y posteriormente se fortalecieron en las universidades, como es el caso de los Programas agrícolas de la Universidad Nacional de Medellín y Palmira y las Universidades de Nariño y Caldas. 
En particular, los inicios de la Educación superior agrícola en la Universidad de Nariño se remontan a 1913, cuando la Asamblea de Nariño fundó el Instituto Agronómico Departamental; sin embargo, algunos inconvenientes no permitieron su desarrollo. Después, en 1959, se constituye el Instituto Tecnológico Agrícola (ITA) y, posteriormente, en 1971, el ITA se transforma en la actual Facultad de Ciencias Agrícolas (FACIA) ${ }^{49}$.

Por lo demás, es indudable la evolución positiva de la Educación agrícola en el país; sin embargo, su futuro no parece despejado y son evidentes sus problemas, e incluso enfrenta retos más desafiantes, como la globalización, los Tratados de Libre Comercio, el deterioro ambiental y el escaso presupuesto. Al respecto, Machado $^{50}$ afirma que el sector rural del país afronta problemas estructurales y destaca que, en los últimos años, la academia ha intervenido en los problemas del sector agropecuario, con la incursión de otras disciplinas, como la Historia, la Sociología y la Ecología, para concebir una Educación agrícola capaz de generar procesos de desarrollo.

\section{HEGEMONÍA DE LAS MISIONES EXTRANJERAS EN LA EDUCACIÓN AGRÍCOLA EN COLOMBIA}

La influencia de las misiones internacionales se dio principalmente en el área de la educación, pero también se dio en otras áreas, como la financiera, en 1923 (Misión Kemmerer), y militar, en 1924, con la Misión Militar Suiza.

En la década de 1920, los colombianos presencian un verdadero desfile de misiones extranjeras. Los distintos gobiernos contratan una misión financiera y administrativa norteamericana dirigida por el economista Edwin W. Kemmerer, una misión penal italiana, una misión agrícola puertorriqueña, una misión telegráfica belga, una misión médica francesa, una misión pedagógica alemana y la misión militar suiza. Además, una misión inglesa trabaja en la red ferroviaria y el Gimnasio Moderno de Agustín Nieto Caballero contrata los servicios del pedagogo belga Ovide Decroly ${ }^{51}$.

Por otra parte, Buriticá ${ }^{2}$ cita un estudio sobre la enseñanza agrícola superior elaborado por la FAO en 1957, que insinúa el predominio de los Estados Unidos en Latinoamérica en el primer cuarto del siglo XX, con la fundación del Colegio de Agricultura y Artes Mecánicas de Puerto Rico, influencia que continuó en aumento en el segundo cuarto de ese siglo; igualmente, la Escuela de Vicosa en

49. Francisco Torres. La educación agrícola en el Departamento de Nariño: Una mirada a la historia y al presente. En: Revista Historia de la Educación en Colombia, № 15 (2012): 206-212.

50. Absalón Machado. La academia y el sector rural (Bogotá: Universidad Nacional de Colombia, 2004 ), 5.

51. Aline Helg. El Desarrollo de la Instrucción Militar en Colombia en los Años 20: Estudio del impacto de una misión militar suiza, 2, [en: Revista Colombiana de Educación, 17 (en.-jun. 1986): 19-40], http:// www. pedagogica.edu.co/storage/rce/articulos/17_05ens.pdf (Septiembre 23 de 2014).

52. Buriticá, Las enfermedades de las plantas y su cienciā en Colombia, 112. 
Brasil (1927) fue un intento de trasplantar y adaptar al medio latinoamericano la orientación de los Land Grant Colleges de Estados Unidos.

Es preciso profundizar un aspecto trascendental que explica por sí solo parte de la situación antes referida, y corresponde al impacto de las misiones extranjeras que se establecieron en el país en diferentes épocas con el objetivo de diagnosticar y recomendar acciones tendientes a redireccionar el sistema educativo colombiano y, en particular, cómo influyeron en los diseños curriculares y los Planes de estudio de los programas agrícolas en Colombia.

De manera cronológica, se examinan a continuación los principales aportes de las misiones extranjeras, que tuvieron un papel determinante, inicialmente como diagnóstico de la situación económica y social de Colombia, y luego diseñaron e implementaron, con la anuencia de los gobiernos de turno, las políticas de la educación agrícola en Colombia mediante la importación de modelos de desarrollo hegemónicos.

\subsection{Misión Pedagógica Alemana}

En la presidencia de Pedro Nel Ospina (1922-1926), y con el propósito de subsanar debilidades del sistema educativo, se propone la Reforma Instruccionista de 1923; para ello se contrató una Misión Pedagógica Alemana, con el objetivo de reformular Planes de desarrollo educativo. Entre otras reformas, se incluyó la gratuidad de la educación primaria y el control de la instrucción pública y, a nivel de Educación Superior, pretendía la centralización de la enseñanza universitaria. Uribe $^{53}$ lo reseña de la siguiente manera:

Quiso la Misión Pedagógica reunir en una sola las cinco Universidades que hoy existen en Colombia, centralizándolas en Bogotá, y dejando a las de Antioquia, Bolívar, Cauca y Nariño como simples Facultades Universitarias, dependientes de la Universidad Central, la cual estaría a cargo de un Rector, y tendría un protector especial, denominado Procurador Universitario.

No obstante, estas pretensiones generaron un amplio debate a nivel nacional, varias recomendaciones no se consumaron y, finalmente, se impidió la centralización de la enseñanza.

\subsection{La Misión Kemmerer}

Una de las misiones extranjeras de mayor impacto en Colombia, a principios del siglo XX, fue la Misión Kemmerer (1923), que se hizo efectiva mediante la Ley 25 de 1923 que dio origen al Banco de la República. Luego, en 1930, el gobierno de Colombia autorizó una segunda Misión Kemmerer, esta vez con el objetivo

53. Antonio Uribe. Política Instruccionista (Bogotá: Imprenta Nacional, 1926), 35. 
de asesorar al Estado en materia de impuestos, deuda externa y precios del café y con el fin de enfrentar la crisis generada por la recesión económica mundial de $1929^{54}$.

Las recomendaciones específicas que se acogieron, en relación con la educación agrícola, tienen que ver directamente con la incentivación de las exportaciones y, en ese sentido, se hizo necesario mejorar la producción agrícola, de allí que el Estado promulgó, entre otras medidas, la Ley de Fomento de la Agricultura, de 1926.

En concepto de Bejarano ${ }^{55}$, la mayoría de los esfuerzos realizados en Colombia hasta 1940, relacionados con el desarrollo agrícola, fueron el resultado de las recomendaciones de estas misiones extranjeras, por ejemplo, el denominado Plan Denemoustier ${ }^{56}$ (1915 y 1917), que creó algunas líneas de experimentación y que, años más tarde, se concretaron en la introducción de pastos y gramíneas de clima frío.

\subsection{La Misión Pedagógica Puertorriqueña}

A partir de 1940, esta misión, dirigida por Lorenzo García, organizó las primeras Escuelas vocacionales agrícolas en Boyacá, Cundinamarca, Nariño y otros Departamentos, acontecimiento que marcó los inicios de la capacitación técnica agrícola en el país.

Al considerar la importancia de la divulgación científica y las estrategias para llevar el conocimiento al sector rural, el gobierno colombiano contrató esta Misión para la capacitación de técnicos agrícolas, mediante cursos de Extensión rural en varias zonas del país. También, otras actividades relacionadas tienen lugar a partir de 1946, cuando el Ministerio de Economía contrató los servicios de Extensión rural para adiestrar ingenieros agrónomos.

Derivado de estas recomendaciones, después de 1950, el Ministerio de Agricultura organizó la División de Extensión Agrícola, y en 1952 el Servicio Técnico Agrícola Colombo Americano (STACA), método que inició actividades en 1954 mediante asesoría del Servicio de Extensión de Costa Rica, y se fundaron en el país las tres primeras Agencias de Extensión en Boyacá, y de manera sucesiva se completaron diez oficinas en distintas partes del país ${ }^{57}$.

54. La Gran Depresión tuvo lugar en los Estados Unidos y se extendió hasta 1940 a la mayoría de países del mundo, a partir de la caída de la Bolsa de Valores de Nueva York.

55. Bejarano, Ensayos de historia agraria colombiana, 190-194.

56. El ministro de Agricultura y Comercio, Jorge Delgado, en la presidencia de José Vicente Concha (19141918), contrató, en 1914, al Ingeniero Agrónomo belga Carlos Denemoustier, con funciones específicas para organizar la enseñanza de las Ciencias agrícolas y formuló el Plan de Educación Agrícola, como eje del actual sistema de educación del sector.

57. Mesa, La agronomía en Colombia, 43-44. 


\subsection{La Misión Currie}

La Misión Currie ${ }^{58}$, de 1949, tenía como objetivo principal diseñar las recomendaciones en torno al desarrollo agrícola del país; en 1951, entregó recomendaciones al gobierno de Colombia, entre otras, sobre el crédito agrícola, la utilización eficiente de la tierra y la coordinación de programas de explotación de la tierra. En efecto, las acciones derivadas de esta legislación afectaron las condiciones de vida de la población rural, lo que incluía, por supuesto, el tema de la educación.

\subsection{Las Fundaciones Rockefeller y Kellogg}

De acuerdo a Roldán ${ }^{59}$, antes de la Segunda Guerra Mundial, la enseñanza y el desarrollo de las ciencias en Colombia evidenciaban una fuerte influencia europea, principalmente de Francia; después del conflicto, la orientación es norteamericana, a partir de los textos, manuales y las relaciones con universidades de Estados Unidos que permitieron la creación de nuevos programas académicos en la Universidad Nacional, como Economía, Geología e Ingeniería Química. De hecho, la influencia estadounidense en la Educación Superior sería notable sólo a partir de los años cincuenta, sobre todo canalizada a través de la Fundación Rockefeller ${ }^{\mathbf{6 0}}$.

El dominio de la Misión Rockefeller, en Colombia, es indiscutible en virtud de un convenio que se firmó con el Ministerio de Agricultura en 1950, cuya sede se ubicó en Agronomía, en la sede de la Universidad Nacional de Medellín. El sistema pretendía controlar la política agraria y la enseñanza agrícola mediante la intervención en los programas curriculares y en los Planes de estudio, lo que finalmente condujo a la creación de varios programas de entomología, fitopatología y mejoramiento de cultivos en varias regiones del país.

Arango ${ }^{61}$ asegura que se pretendía implantar un modelo de enseñanza similar a los Land Grand Colleges, que se fundamentaba en un elevado uso de insumos, mínimo empleo de mano de obra, la genética y el control químico de las malezas; después, el dominio tiene, como estrategias, la cooperación de financiera y técnica de las Fundaciones Rockefeller y Kellogg mediante programas de especialización

58. Decsi Arévalo. Misiones económicas internacionales y política en Colombia 1930-1960 (Bogotá: Universidad de los Andes, 1997), http://historiacritica.uniandes.edu.co/view.php/462/view.php (mayo 27 de 2014).

59 Diego Roldán. El perfil y la formación del profesional en ciencias agropecuarias y afines (Bogotá: TM Editores/COLCIENCIAS/AGROFUTURO, 1999), 5.

60. Bejarano, Ensayos de historia agraria colombiana, 188.

61. Mario Arango. La revista Facultad Nacional de Agronomía, Medellín como indicador del fraccionamiento especializado del saber agronómico. En: Revista Facultad Nacional de Agronomía. Medellín: Vol. 58, No. 2 (2005): 2804-2806. 
de profesores colombianos en los Estados Unidos, contratos cooperativos con universidades norteamericanas y otras formas menos notorias de difusión de su cultura agrícola. Un aspecto central de la influencia de los Estados Unidos fue la adopción del esquema de educación investigación-extensión, característico de los colegios agrícolas de ese país.

\subsection{La Misión Lebret}

El gobierno militar de Gustavo Rojas Pinilla (1953-1957) contrató, en 1954, un estudio con un grupo de profesionales extranjeros, dirigido por Louis-Joseph Lebret; ${ }^{62}$ su informe final revela, entre otros resultados, que el desarrollo económico del país sólo beneficiaba a los ricos, y el acceso a la educación para la población pobre era difícil; de allí, también, se derivan otras recomendaciones relacionadas con reformas educativas y apertura democrática, para evitar que el proceso de desarrollo del país se detuviera ${ }^{63}$.

En Colombia, el período comprendido entre 1953 a 1957 fue de intensas connotaciones políticas, económicas y sociales, y se hizo evidente la influencia de las misiones extranjeras. En el campo político, de destacan la Contrarreforma conservadora, el asesinato de Jorge Eliécer Gaitán, el golpe militar de Gustavo Rojas Pinilla y la instauración del Frente Nacional. En el aspecto económico, se acentúan los diagnósticos socioeconómicos de las misiones extranjeras, el Banco Internacional de Reconstrucción y Fomento (BIRF) y el Banco Mundial, diagnósticos que precedieron algunos procesos, como el Primer Plan Quinquenal de educación y Plan decenal de economía, además del rápido crecimiento de las ciudades, el desarrollo industrial y la ampliación de cobertura de la educación primaria, secundaria y superior ${ }^{64}$.

El estudio desplegado por la Misión Lebret ${ }^{65}$, en un extenso reporte entregado al Estado colombiano, reconocía primordialmente a la educación agrícola como estrategia para impulsar el desarrollo, y hacía recomendaciones concretas en torno a la capacitación de técnicos y profesionales agrícolas para aprovechar óptimamente el suelo mediante el fortalecimiento de los programas académicos en universidades del país.

62. Louis-Joseph Lebret, economista y religioso de la orden dominicana, que nació en Francia en 1897 y murió en París en 1966.

63. Albeiro Valencia. Los programas de maestría y doctorado en el marco de la Reforma de la Educación Superior en Colombia. En: Diana Soto, Manuel Lucena y Carlos Rincón (eds.) Estudios sobre la universidad latinoamericana, de la Colonia al siglo XXI (Tunja: Universidad Pedagógica y Tecnológica de Colombia/ RUDECOLOMBIA/COLCIENCIAS, 2010), 333.

64. Alba Nidia Triana. La educación rural en Colombia: De la Contrarreforma conservadora al Frente Nacional (Tunja: Búhos Editores, 2008), 21.

65. La Misión Lebret, “Misión Economía y Humanismo”, entre diciembre de 1954 y junio de 1956, desarrolló en varias regiones del país el trabajo: Estudio sobre las condiciones del desarrollo de Colombia. 
Por otro lado, el informe destacaba el poco interés del Estado en desarrollar este campo de la educación y aseguraba: "La enseñanza profesional de los jóvenes, de la cual se trata aquí, no tiene todavía un lugar importante dentro del esfuerzo educacional en Colombia"66.

No obstante, durante el período de 1955 hasta 1964, y como resultado de la influencia directa de los organismos internacionales, se observa en el país una inusitada actividad de apertura de programas de educación agrícola, entre los que se destacan los programas de la Universidad del Tolima, la Universidad de Nariño, la Universidad de Caldas y la Universidad Tecnológica del Magdalena, entre otros.

\subsection{Alianza para el Progreso}

La Conferencia celebrada en Punta del Este (Uruguay), en 1961, con participación de los países de la Organización de Estados Americanos (OEA), aprobó la creación de la Alianza para el Progreso (ALPRO), y estableció, entre otros objetivos, mejorar la vida de los habitantes del continente y, para ello, se proclamaron varias acciones de carácter social (educación, sanidad y vivienda) que les exigían a sus miembros la organización de programas nacionales de desarrollo económico y social, con el máximo empleo de los recursos naturales ${ }^{67}$.

Otros aspectos de connotación económica y social, que influyeron en el direccionamiento de políticas gubernamentales para el sector agrícola en Colombia, resultaron de las acciones directas de la Alianza para el Progreso, como plataforma de ayuda económica, política y social de Estados Unidos para América Latina. Esta propuesta de índole hemisférica surgió por iniciativa del presidente John F. Kennedy y se proyectó a cuatro años, con una inversión de 2000 millones de dólares. Según apreciación de $\mathrm{Helg}^{68}$, los dirigentes colombianos de la época, por el "orgullo de pertenecer al concierto mundial", aceptaron compromisos de carácter financiero y técnico, en su propósito de obtener créditos externos.

Estas circunstancias, a nivel interno y externo, contribuyeron a fortalecer los programas de Educación Superior encaminados a mejorar la producción agrícola nacional; sin embargo, Valencia ${ }^{69}$ argumenta que las estrategias de la Conferencia de Punta del Este se priorizaron para impedir el avance de la Revolución Cubana, y concluye lo siguiente:

66. Louis-Joseph Lebret. Estudio sobre las condiciones del desarrollo de Colombia (Bogotá: Presidencia de la República/Comité Nacional de Planeación/ Editores Cromos, 1958), 311.

67. Carlos Sanz de Santamaría. El estado financiero de la nación. En: Jorge Vélez García (ed.). Devaluación 1962, Documental de un proceso económico (Bogotá, Ediciones Tercer Mundo, 1963), 45.

68. Helg, La educación en Colombia 1918-1957, 231.

69. Albeiro Valencia. Los programas de maestría y doctorado en el marco de la reforma de la educación superior en Colombia. En: Diana Soto, Manuel Lucena y Carlos Rincón (eds.). Estudios sobre la universidad latinoamericana, de la Colonia al siglo XXI (Tunja: Búhos Editores, 2010), 334. 
El gobierno del presidente John F. Kennedy se preparó con la Alianza para el Progreso y con los Cuerpos de Paz. Y en lo que tenía que ver con la Educación Superior, el informe del experto Rudolph Atcon fijó las pautas de modernización y racionalización para las universidades latinoamericanas. En nuestro país, la educación empezó a ser influenciada política y financieramente por los Estados Unidos. Durante los años 1960 a 1967 el país recibió 48 millones de dólares para el fomento de la educación, de los cuales 28 millones se invirtieron en Educación Superior, para construcción de edificios y desarrollo de nuevos programas ${ }^{\mathbf{7 0}}$.

\section{CONCLUSIONES}

La instauración y fortalecimiento de la educación agrícola a todo nivel, pero principalmente a nivel superior, se ata históricamente a los procesos de desarrollo del país desde la época republicana, y las influyen misiones europeas desde principios del siglo XX. Después de la Segunda Guerra Mundial, se hace evidente el dominio de las Misiones de Estados Unidos en su clara intención de propiciar el uso intensivo de insumos agroquímicos, mediante el modelo inspirado en la Revolución Verde, que pretendía inicialmente el incremento de los rendimientos por unidad de producción, especialmente en cereales; sin embargo, sus efectos nocivos sobre el medio ambiente han propiciado un rechazo generalizado.

En este sentido, los cambios y ajustes de las políticas educativas del Estado a lo largo de su Historia los han trastocado acontecimientos, tanto internos como externos, con incontables reformas, leyes, decretos, misiones y convenios, que han cimentado el marco legal sobre el que se han establecido varias Facultades de Ciencias Agrícolas en todo el territorio nacional, y su evolución es significativa; no obstante, sus efectos sobre el desarrollo del país dejan serias dudas sobre su efectividad y su futuro no parece despejado, y es evidente, por lo demás, la agudización de la problemática agraria, el abandono del campo y la contaminación ambiental.

Ante estos escenarios poco abordados por los investigadores y, por lo demás, preocupantes, descritos y analizados a la luz de acontecimientos históricos y actuales, es urgente estimular, en la comunidad académica, los gremios de la producción y los dirigentes políticos y sociales, reflexiones, debates y acciones concretas en torno a los currículos agrícolas y el marco del desarrollo para propiciar el diseño de modelos más equitativos, que examinen la realidad, donde la Educación agrícola constituya una verdadera opción de desarrollo liberador de la población rural.

70. Valencia, “Los programas de maestría y doctorado en el marco de la Reforma de la Educación Superior en Colombia, 334. 


\section{REFERENCIAS BIBLIOGRÁFICAS}

Agudelo, Hernando. Cuatro etapas de la inflación en Colombia. Bogotá: Tercer Mundo, 1966.

Alarcón, Pedro y Néstor Romero. Estado actual de los currículos de Ingeniería Agronómica y Agronomía en Colombia (Bogotá: ACOFIA, 2013), api.ning.com/files/.../librocompleto.docx.

Arango, Mario. La Revista Facultad Nacional de Agronomía, Medellín como indicador del fraccionamiento especializado del saber agronómico. En: Revista Facultad Nacional de Agronomía. Vol. 58. No. 2 (2005): 2801-2811.

Arévalo, Decsi. Misiones económicas internacionales y política en Colombia 1930-1960. Bogotá: Universidad de los Andes, 1997, http://historiacritica.uniandes.edu.co/view.php/462/view.php (mayo 27 de 2014.

Banco Mundial. El Desarrollo Económico de Colombia, Problemas y perspectivas. Bogotá: Biblioteca Banco Popular, 1970.

Bejarano, Jesús A. Ensayos de historia agraria colombiana. Bogotá: Fondo Editorial CEREC, 1987.

Bejarano, Jesús. Economía de la Agricultura. Bogotá: TM Editores/Universidad Nacional/IICA, 1998.

Buriticá, Pablo. Las enfermedades de las plantas y su ciencia en Colombia. Medellín: Universidad Nacional de Colombia, 1999.

De Pombo, Joaquín. Dinámica del sector agrícola colombiano (pp. 210-230). En: Francisco Thoumi et al (eds.). La economía colombiana, temas de comercio y desarrollo. Bogotá: Banco de la República, 1995.

Duarte, Jesús. Educación pública y clientelismo en Colombia. Medellín: Facultad de Ciencias Humanas y Económicas/Universidad Nacional de Colombia/Sede Medellín, 2003.

González, Silvia. Historia de la Hacienda de Chapingo. México: Universidad Autónoma de Chapingo, 1996.

Goyes, Isabel y Mireya Uscátegui. Investigación y pedagogía. Pasto: Universidad de Nariño, 1999.

Gracia, Roberto. La educación agrícola vocacional en Colombia (IV B, pp. 1-22). En: Gerardo Naranjo (ed.). La educación agrícola para el desarrollo social y económico. Bogotá: Instituto Interamericano de Ciencias Agrícolas (OEA)/Instituto para el Fomento de la Educación Superior (ICFES)/ Programa de Estudios para Graduados Universidad Nacional de Colombia/ICA, 1977.

Helg, Aline. El Desarrollo de la Instrucción Militar en Colombia en los Años 20: Estudio del impacto de una misión militar suiza, 2, [en: Revista Colombiana de Educación, 17 (en.-jun. 1986): 19-40], http://www. pedagogica.edu.co/storage/rce/articulos/17_05ens.pdf (septiembre 23 de 2014).

Helg, Aline. La educación en Colombia 1918-1957. Bogotá: Fondo Editorial CEREC, 1987.

Instituto Técnico Agrícola. Reseña histórica. Instituto Técnico Agrícola de Guadalajara de Buga (Valle del Cauca), http://www.ita.edu.co/index.php/2013-10-18-14-31-32/resena-historica (14 de abril de 2013).

Lebret, Louis-Joseph. Estudio sobre las condiciones del desarrollo de Colombia. Bogotá: Editores Cromos, 1958.

León, Tomás. Medio ambiente, tecnología y modelos de agricultura en Colombia. Bogotá: Ecoe Ediciones, 2007.

Machado, Absalón. La academia y el sector rural. Bogotá: Universidad Nacional de Colombia, 2004.

Macías, Daniel. 50 años de la Agronomía en Colombia. Bogotá: Duplolito, 1978.

Mesa, Daniel. La agronomía en Colombia. Bogotá: III Congreso Nacional de Ingenieros Agrónomos (s.f).

Molina, Luis Fernando. Salvador Camacho Roldan. Bogotá: Biblioteca Virtual Luis Ángel Arango del Banco de la República, http://www.banrepcultural.org/blaavirtual/biografias/camasal.htm (septiembre 23 de 2013).

Ocampo, Javier (2002). Los orígenes oficiales de las universidades republicanas en la Gran Colombia (18261830). En: Diana Soto, Manuel Lucena y Carlos Rincón (eds.). Estudios sobre la universidad latinoamericana, de la Colonia al siglo XIX. Tunja: Búho Editores/Universidad Pedagógica y Tecnológica de Pereira, 2002. 
Ovalle, Julio. Expedición Botánica: Revelaciones del Nuevo Mundo. Bogotá: Biblioteca Nacional de Colombia: Exposiciones Virtuales, 2008, http://www.bibliotecanacional.gov.co/recursos_user/exposiciones virtuales/ expedicion_botanica/pdf/expedicion_botanica.pdf (23 de Julio de 2012).

Patiño, Víctor. Esbozo histórico agropecuario del periodo republicano en Colombia. Bogotá: Instituto Caro y Cuervo, 2002.

Roldán, Diego. El perfil y la formación del profesional en ciencias agropecuarias y afines. Bogotá: TM Editores/ COLCIENCIAS/AGROFUTURO, 1999.

Sanz de Santamaría, Carlos. El estado financiero de la nación (pp. 38-51). En: Jorge Vélez García (ed.). Devaluación 1962, Historia documental de un proceso económico. Bogotá: Ediciones Tercer Mundo, 1963.

Suárez, Aurelio. El modelo agrícola colombiano y los alimentos en la globalización. Bogotá: Ediciones Aurora, 2007.

Torres, Francisco. La educación agrícola en el Departamento de Nariño: Una mirada a la Historia y al presente. En: Revista Historia de la Educación en Colombia, No. 15 (2012): 193-214.

Triana, Alba Nidia. Escuelas Normales rurales, agropecuarias y de campesinas en Colombia: 1934-1974. En: Revista Historia de la Educación Colombiana, No. 13 (2010): 201-230.

Triana, Alba Nidia. Formación de maestros rurales colombianos 1946-1994. En: Revista Historia de la Educación Latinoamericana. Vol. 14. No. 18 (2012): 93-118.

Triana, Alba Nidia. La educación rural en Colombia: De la Contrarreforma conservadora al Frente Nacional. Tunja: Búhos Editores, 2008.

Uribe, Antonio. Política Instruccionista. Bogotá: Imprenta Nacional, 1926.

Uscategui, Mireya. La institucionalización de la enseñanza del arte en Colombia: Antecedentes y evolución (De la Colonia al siglo XIX). Pasto: Universidad de Nariño/RUDECOLOMBIA, 2010 (Tesis Doctoral).

Valencia, Albeiro. Los programas de maestría y doctorado en el marco de la Reforma de la Educación Superior en Colombia (pp. 331-343). En: Diana Soto, Manuel Lucena y Carlos Rincón (eds.). Estudios sobre la universidad latinoamericana, de la colonia al siglo XXI. Tunja: Búhos Editores, 2010.

Valencia L., Néstor. Génesis e irrupción de la agricultura científica: La educación agrícola superior en el Valle del Cauca, Colombia, 1910-1946. Pereira: Universidad Tecnológica de Pereira/RUDECOLOMBIA, 2006 (Tesis Doctoral).

Valencia, Néstor y Álvaro Acevedo. Políticas agrarias del Estado-nación colombiano. Los inicios de la educación agrícola superior en el Valle del Cauca (pp. 213-231). En: Diana Soto, Justo Cuño, Jorge Tomas y Jorge Acuña (eds.). La construcción de la Nación Iberoamericana. Siglos XIX a XXI. Bogotá: VENDIMIA, 2010. 